\title{
DES CURÉS CHEZ BACCHUS. SATIRE ANTICLÉRICALE ET OPPOSITION POLITIQUE CHEZ GUSTAVE COURBET (1863-1868)
}

Bertrand Tillier

Armand Colin | « Romantisme »

2013/4 n 162 | pages 59 à 72

ISSN 0048-8593

ISBN 9782200928858

Article disponible en ligne à l'adresse :

http://www.cairn.info/revue-romantisme-2013-4-page-59.htm

\section{Pour citer cet article :}

Bertrand Tillier, « Des curés chez Bacchus. Satire anticléricale et opposition politique chez Gustave Courbet (1863-1868) », Romantisme 2013/4 ( $\mathrm{n}^{\circ} 162$ ), p. 59-72.

DOI 10.3917/rom.162.0059

Distribution électronique Cairn.info pour Armand Colin.

(C) Armand Colin. Tous droits réservés pour tous pays.

La reproduction ou représentation de cet article, notamment par photocopie, n'est autorisée que dans les limites des conditions générales d'utilisation du site ou, le cas échéant, des conditions générales de la licence souscrite par votre établissement. Toute autre reproduction ou représentation, en tout ou partie, sous quelque forme et de quelque manière que ce soit, est interdite sauf accord préalable et écrit de l'éditeur, en dehors des cas prévus par la législation en vigueur en France. Il est précisé que son stockage dans une base de données est également interdit. 


\section{Des curés chez Bacchus. Satire anticléricale et opposition politique chez Gustave Courbet (1863-1868)}

C'est à la lumière de la guerre d'escarmouches conduite contre Napoléon III et le Second Empire par Gustave Courbet, désireux d'établir son statut d'artiste émancipé ${ }^{\text {, }}$ qu'il faut considérer les deux plaquettes illustrées, Les Curés en goguette ${ }^{2}$ et La Mort de Jeannot. Les Frais du culte ${ }^{3}$, publiées par le peintre "à l'appui ${ }^{4}$ " de deux de ses tableaux anticléricaux, Le Retour de la conférence (également appelé Les Curés ou La Soûlerie) et La Mort de Jeannot à Ornans (dit aussi Le Vol). Aujourd'hui disparues, ces deux toiles avaient été envoyées au Casino de Gand, avec onze autres, pour une exposition organisée du 3 septembre au 15 novembre 1868. De même que l'érotique Femme nue au chien, elles furent écartées de l'exposition ordinaire et accrochées dans une salle séparée, fermée au grand public ${ }^{5}$. Depuis Le Havre où il séjourne, Courbet écrit au secrétaire de la Société royale d'encouragement des Beaux-Arts, en charge du Salon de Gand :

[...] j'ai cru comprendre que nous avions quelque chose à faire pour lutter contre Messieurs les curés, j'ai pensé que c'était d'autant plus utile qu'on continuait à crucifier à Anvers. Vous avez mes tableaux, l'un Le Vol, l'autre La Soûlerie ${ }^{6}$. À l'appui de ces deux tableaux j'ai fait écrire deux notices, ou histoires de faits véridiques à ma connaissance qui ont donné lieu à ces tableaux. J'espère que ça va donner lieu à une grande guerre, car j'ai dans la volonté l'idée de les pousser loin ${ }^{7}$.

Le peintre ajoute: "Nous allons foutre les curés à la solde de ceux qui les emploieront, ça sera honnête pour eux d'abord et pour nous ensuite, car il faut que ça finisse ${ }^{8}$." Dans ce contexte, Courbet diffuse ses deux opuscules et dix reproductions

1. Bertrand Tillier, « Courbet, un utopiste à l'épreuve de la politique », catalogue de la rétrospective Gustave Courbet, Paris, Grand Palais, 2007/Montpellier, musée Fabre, 2008, p. 19-28.

2. Les Curés en goguette, avec six dessins de Gustave Courbet, Exposition de Gand de 1868, Bruxelles, A. Lacroix, Verboeckhoven et Cie éditeurs, 1868.

3. La Mort de Jeannot, Les Frais du culte, avec quatre dessins de Gustave Courbet, Exposition de Gand de 1868, Bruxelles, A. Lacroix, Verboeckhoven et Cie éditeurs, 1868.

4. Dans Correspondance de Courbet, éd. de Petra ten-Doesschate Chu, Paris, Flammarion, 1996, p. 303 (à Alfred Bruyas, 10 septembre 1868).

5. Jörg Zutter et Petra ten-Doesschate Chu, Courbet, Artiste et promoteur de son auvre, Paris, Flammarion, 1998, p. 106.

6. Soit La Mort de Jeannot à Ornans et Le Retour de la conférence. 1868).

7. Correspondance de Courbet, ouvr. cité, p. 303-304 (à Ferdinand van der Haeghen, 11 septembre

8. Ibid., p. 304. 
photographiques de ses illustrations s'y rapportant, qu'il vend séparément. Il précise aux organisateurs : "Il faudra mettre à proximité un registre où pourraient s'inscrire les personnes qui désireraient avoir ou cette collection, ou les photographies séparées ${ }^{9}$. " De ces modes d'intervention politique et polémique que sont le pamphlet et l'image satirique, où Courbet convoque l'arme de l'anticléricalisme, seul l'opuscule des Curés en goguette, pour lequel des sources sont accessibles, retiendra ici notre attention.

\section{UN TABLEAU D'OPPOSITION}

Par sa brochure de 1868, Courbet entend relancer la campagne irréligieuse qu'il avait lancée cinq ans plus tôt avec Le Retour de la conférence, "tableau capital » et " le plus grotesque qu'on aura jamais vu en peinture " - «tableau de curés ${ }^{10}$ ", comme il le nomme dans une expression elliptique qui dit bien l'économie satirique et anticléricale -, "tableau critique et comique au dernier degré ${ }^{11}$ ". L'œuvre représente, sur un chemin de Franche-Comté, un cortège de sept curés ivres - " comme les sept vaches grasses de la Bible ou les sept pêchés (sic) capitaux ", note Castagnary ${ }^{12}-$, suivis de leurs servantes, de retour d'une conférence ecclésiastique ${ }^{13}$, tenue chaque lundi, à tour de rôle, chez chacun des prêtres du doyenné d'Ornans. On y agite des questions de religion, de morale et d'histoire ; souvent, quand « le tempérament des convives s'y prête et quand les finances de l'hôte le permettent, suit un "plantureux" dîner, abondant en vins généreux et en bonne chère ", comme l'explique Georges Riat $^{14}$. Dans le texte que le peintre avait commandé à Proudhon pour en faire une brochure d'accompagnement de son tableau ${ }^{15}$, et dont le philosophe tirera finalement un traité sur l'art, l'iconographie de l'œuvre est soigneusement décrite :

Au premier plan on voit un groupe de quatre prêtres, dont l'un, incapable de traîner sa vaste corpulence, a été hissé sur un âne, qui plie sous le faix. C'est le doyen ; [...] son front soucieux, ses lèvres lippues, son œil en ce moment quelque peu lubrique, son port de Silène décèlent un joyeux convive [...]. Il est soutenu à gauche $[. .$.$] par un jeune vicaire [...], bellâtre montagnard, giton de sacristie,$ miroir à dévotes, cherchant avant tout, dans la carrière ecclésiastique, les joies du bien-être [...]. À droite est un curé d'âge mûr, mais vif et vert, à lunettes bleues, au

\section{Ibid.}

10. Ibid., p. 193 (à Léon Isabey, décembre 1862).

11. Ibid., p. 195 (à ses parents, début janvier 1863).

12. Jules Castagnary, Salons, 2 vol., Paris, Charpentier et Fasquelle, 1892, vol. I, p. 149.

13. Les conférences ecclésiastiques, dites aussi cantonales, réunissaient, en principe une fois par mois, les desservants d'un même canton chez le curé-doyen résidant au chef-lieu de canton, pour une journée de réflexion, de travail intellectuel et de préparation des prédications, sur un thème défini par l'évêché (Évangiles, théologie dogmatique, morale ou pastorale). Voir Marcel Launay, Le Bon prêtre. Le clergé rural au XIX ${ }^{e}$ siècle, Paris, Aubier, « collection historique », 1986, p. 59-61. Je remercie Jacqueline Lalouette des précisions qu'elle m'a permis d'apporter dans cet article sur les points relatifs à la question religieuse.

14. Georges Riat, Gustave Courbet peintre, Paris, H. Floury, coll. « Les maîtres de l'art moderne », 1906, p. 201-202.

15. «En ce moment je suis en correspondance avec Proudhon. Nous faisons ensemble un ouvrage important qui rattache mon art à la philosophie et son ouvrage au mien. C'est une brochure qui sera vendue dans mon exhibition en Angleterre [...] » (Correspondance de Courbet, ouvr. cité, p. 205, à son père, 28 juillet 1863). 
teint bilieux, figure de fouine ou de diplomate, Talleyrand rustique, qui retient par le bras le Silène chancelant. Prudent et expérimenté, il comprend les inconvénients du scandale, et voudrait sauver au moins les apparences. [...] À l'abri de l'âne est un abbé de bon ton, l'hôte du château et des bonnes maisons du pays, [...] confesseur de comtesses, ecclésiastique du monde à destination spéciale, aspirant évêque. La médisance ne l'atteint pas encore ; il baisse la tête, comme s'il voulait se dérober aux regards, et s'efforce, en entraînant l'âne rebelle, d'abréger ce voyage, qui met sa pudeur au supplice. Derrière ce groupe marche un séminariste, à la figure candide, plein d'une ferveur juvénile, [...] et qui rêve le martyre. [...] Il soutient avec une sollicitude pleine de charité un vieil ecclésiastique trébuchant et frappant la terre de sa canne [...]. À côté d'eux, [...] s'avance carrément un curé d'un type à part : c'est le prêtre herculéen, taillé à angle droit, terrible de visage, admiré des paysans pour la rudesse de ses allures, buvant, fumant et jurant, exerçant sur ses paroissiens un ascendant irrésistible par son énergique vulgarité. [...] Ce n'est qu'un pauvre curé de campagne dont la fougue s'exhale dans des affaires de maquignonnage ou d'insipides conférences [...]. À distance respectueuse vient le groupe des servantes, auxiliaires de la cuisine du banquet, et qui rapportent sur leurs têtes, dans des paniers, quelques bons restes pour le déjeuner du lendemain ${ }^{16}$.

Proudhon précise ensuite: "Toute cette troupe passe devant un vieux hêtre, dans une excavation duquel est placée sous une grille une statuette de la Vierge immaculée ${ }^{17}$. " Il ajoute encore :

Tout à fait à gauche du tableau, et comme pour en exprimer la moralité, se trouvent un paysan et sa femme piochant la terre au bord de la route [...] et distraits un moment de leur travail par le spectacle auquel ils assistent. Le paysan, grossier, illettré, n'en est pas moins de son époque [...]. À la vue des saints hommes en goguette, devant ce foudroyant contraste entre la spiritualité présomptueuse du ministre de l'autel et la réalité bachique de son existence, il est pris d'un rire fou [...] Quant à la femme du paysan, dominée par les enseignements de son enfance, [...] mais douloureusement affectée par ce dont elle est témoin, elle prie Dieu de pardonner à ses fragiles ministres $[\ldots]^{18}$.

Cette œuvre, Courbet l'a conçue, loin de Paris, moins dans le secret que dans le retrait d'une Saintonge ultra-bonapartiste mais profondément anticléricale ${ }^{19}$, où des amis républicains - Jules Castagnary ${ }^{20}$, Étienne Baudry ${ }^{21}$, Théodore Duret ${ }^{22}$ -

16. Pierre-Joseph Proudhon, Du principe de l'art et de sa destination sociale, Dijon, Les Presses du réel, 2002, p. 166-168.

17. Ibid., p. 168.

18. Ibid., p. 168-169.

19. Roger Bonniot, Gustave Courbet en Saintonge, [1973], Cozes, La Saintonge littéraire, 1986, p. $136-137$.

20. Avocat, opposant républicain et critique d'art, Jules Castagnary (1830-1888) se lie à Courbet en 1860 et sera l'artisan de sa réhabilitation après la Commune de 1871.

21. Étienne Baudry (1830-1908) est un riche propriétaire foncier et un amateur d'art qui, par l'entremise de Castagnary, accueillit Courbet en 1862 dans son château de Rochemont, en Saintonge.

22. Homme politique et journaliste, Théodore Duret (1838-1927) est un opposant républicain, fondateur du journal La Tribune. Critique d'art, il compte parmi les premiers défenseurs de Manet et des impressionnistes. 
et leurs réseaux l'ont accueilli. C'est un "tableau d'opposition ${ }^{23}$ ", exécuté pendant la campagne des élections législatives de 1863, en vue du Salon où Courbet aspire surtout à le voir refusé, pour mettre le régime impérial dans l'embarras, susciter le scandale et en retirer des bénéfices politiques et financiers.

J'avais voulu savoir le degré de liberté que nous accorde notre temps. J'avais envoyé un tableau de curés, bien senti : le Retour d'une Conférence. Ça correspondait pas mal avec l'insulte que l'empereur m'a faite l'an passé, d'autre part avec ce qui se passe vis-à-vis des cléricaux. Le tableau a porté juste, est allé droit à son auteur. Il a été dépendu et rependu trois ou quatre fois. En parlant à Walewski on pourrait peut-être l'accrocher une cinquième fois. J'avais fait le tableau pour qu'il soit refusé. J'ai réussi ${ }^{24}$.

Sa correspondance du printemps 1863 montre que Courbet craint de voir le tableau accepté au Salon des Refusés - une décision qui aurait ruiné le programme de son "opération ${ }^{25}$ " combinant une exposition privée dans son atelier, une tournée nationale et internationale dans un barnum et la diffusion d'une reproduction de l'œuvre par le photographe Bingham. L'œuvre sera finalement écartée du Salon des Refusés non pour "insuffisance de peinture ${ }^{26}$ " comme Le Déjeuner sur l'herbe de Manet, mais pour immoralité. L'artiste peut alors mettre en branle une partie de son projet, comme l'attestent les contrats qu'il signe avec les entrepreneurs Félix Ratier et Charles Bataille, puis les marchands Jules Luquet et Alfred Cadart, pour que son tableau voyage en Grande-Bretagne, Suisse, Belgique et Allemagne, et jusqu'à New York: "Mon tableau des Curés part pour l'Amérique, ainsi que Les Lutteurs ", écrit-il avec satisfaction à ses parents ${ }^{27}$, en dévoilant une association d'œuvres très symbolique.

Si Courbet est tant attaché à ce que son Retour de la conférence soit perçu comme doublement refusé et censuré - il feindra de regretter que les reproductions photographiques de son œuvre aient été interdites de vente, saisies et détruites ${ }^{28}$-, c'est parce qu'il l'a conçu comme un " tableau-gifle ${ }^{29}$ " que l'Empereur doit recevoir « en pleine figure $^{30}$ ». Cette œuvre satirique et rabelaisienne, dont la critique a d'emblée souligné la gauloiserie, est une machine de guerre politique, dont Proudhon a montré qu'elle entendait subvertir la morale religieuse comme ordre politique et social établi. Après le refus de son tableau au Salon de 1863, il semble, d'après Georges Riat, que Courbet ait décidé de riposter en composant bientôt deux autres toiles : Le Dîner de la conférence - "dans la discussion, un curé [y] jetterait ses confrères par la fenêtre "et Le Coucher de la conférence, où des servantes seraient montrées couchant les curés

23. Correspondance de Courbet, ouvr. cité, p. 195 (à ses parents, début janvier 1863).

24. Ibid., p. 199 (à Albert de La Fizelière, 23 avril 1863).

25. Ibid., p. 201 (à Champfleury, mi-juin 1863).

26. Ibid., p. 199-200 (à Léon Isabey, fin avril 1863).

27. Ibid., p. 242 (à ses parents, 3 janvier 1866).

28. «La police vient de détruire arbitrairement les clichés des Curés chez Bingham, je ne sais de quel droit [...]. » (Ibid., p. 275, à Jules Castagnary, 21 avril 1867).

29. Selon l'expression de Chakè Matossian, Saturne et le sphinx, Proudhon, Courbet et l'art justicier, Genève, Droz, 2002, p. 122.

30. Correspondance de Courbet, ouvr. cité, p. 199 (à Léon Isabey, avril 1863). 


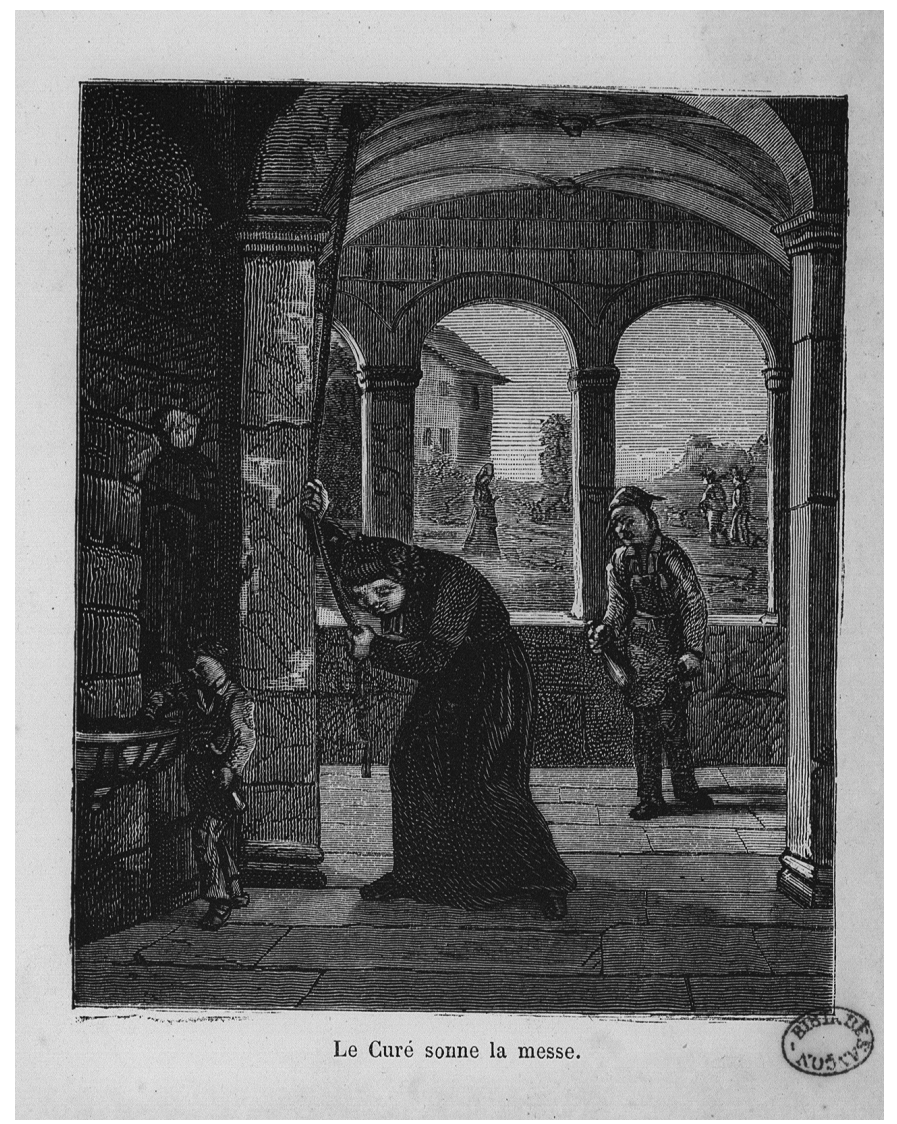

Figure 1. (C) Bibliothèque municipale de Besançon + BR.108.23.

et leur préparant une tisane $\mathrm{e}^{31}$. Or, si l'artiste paraît avoir abandonné ce projet d'une suite de tableaux dont sa correspondance ne fait d'ailleurs pas état, il en reprend sans doute le dessein dans la série des illustrations de sa brochure de 1868. Parce qu'ils ont généralement été jugés "médiocres : lourds, noirs, manquant de perspective, assez inexpressifs ${ }^{32}$ ", les six dessins de Courbet n'ont guère retenu l'attention des historiens de l'art, qui les ont considérés comme les annexes du Retour de la conférence, longtemps vu comme une image plus qu'une œuvre, une caricature plus qu'un tableau.

\section{UNE SUITE EN IMAGES}

En amont et en aval du Retour de la conférence, les illustrations des Curés en goguette racontent en six "stations » le calvaire inversé d'une journée de conférence

31. Georges Riat, Gustave Courbet peintre, ouvr. cité, p. 205.

32. Ibid., p. 264. 


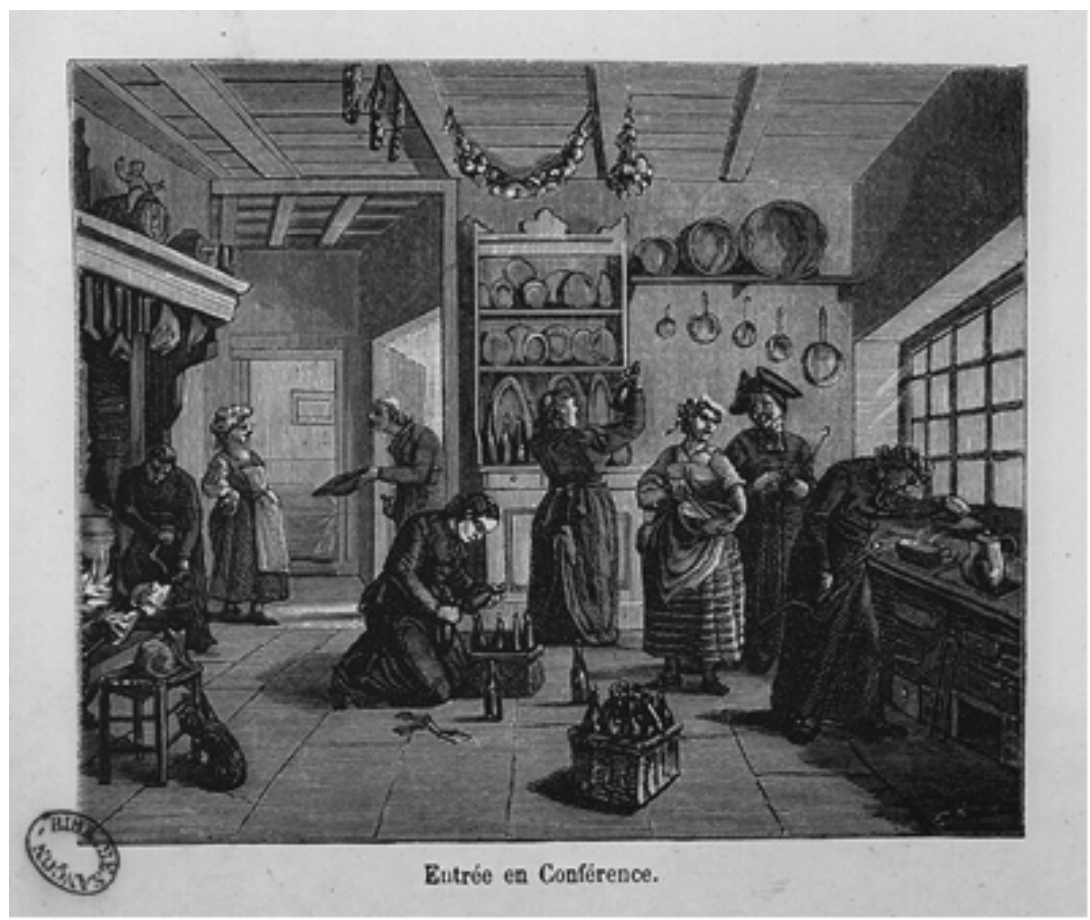

Figure 2. (C) Bibliothèque municipale de Besançon + BR.108.23.

qui « commence à midi et se termine à cinq ou six heures du soir ${ }^{33}$ ", en n'étant que prétexte à ripaille et " distraction pantagruélique ${ }^{34}$ ». Le texte anonyme composant la brochure - Courbet en fut-il le commanditaire ou l'auteur ? -, d'une conception très littérale, est une ekphrasis de chacune des images et d'autres encore que l'artiste aurait pu ajouter, inspirée de la littérature physiologique très en vogue sous la monarchie de Juillet et augmentée d'une dimension pamphlétaire contre les « ignorantins ${ }^{35}$ " que sont les curés soumis à "la lecture obligatoire d'un livre débilitant nommé bréviaire " Dès les premières pages de son opuscule, Courbet s'en prend à la hiérarchie diocésaine, gouvernement et police des populations rurales qui subissent sa tyrannie, reposant sur une fausse spiritualité et une hypocrisie sociale : "Il s'agit [...] de faire connaître ici les faits et gestes de quelques joyeux compères ressemblant plus à des disciples d'Épicure qu'à des serviteurs de celui qui n'avait souvent pas une pierre pour lui servir d'oreiller ${ }^{37}$. " Courbet compose donc une chronique illustrée de la vie du clergé des

33. Les Curés en goguette, ouvr. cité, p. 7.

34. Ibid., p. 6.

35. Les Curés en goguette, ouvr. cité, p. 6. Le terme «ignorantins » s'appliquait aux Frères des écoles chrétiennes. Courbet l'emploie improprement, sans doute dans un esprit anticlérical et satirique, pour railler l'ignorance des curés.

36. Ibid., p. 5.

37. Ibid.. Cette phrase fait référence à un verset de l'évangile de saint Luc $(9,58)$ « Les renards ont des terriers et les oiseaux du ciel des nids ; le Fils de l'homme, lui, n'a pas où poser la tête. » 
campagnes, à l'aide de scènes réalistes, au sens où il a pu définir cette esthétique : " un art essentiellement concret [qui] ne peut consister que dans la représentation des choses réelles et existantes [...] une langue toute physique ${ }^{38}$."

Dès la première planche [fig. 1], Courbet feint d'opposer deux conceptions du monde : celle de la vie sociale - à l'arrière-plan, dans la trouée de l'architecture, «les chasseurs se mettent en route pour la bécasse, suivis de leurs chiens d'arrêt en grelots " - et celle de la vie (faussement) pieuse : au premier plan, une bigote difforme se tient près du bénitier où « le curé sonne la messe ", mais le sacristain " accourt, muni d'une bouteille pleine d'un merveilleux liquide ${ }^{39}$ ». Dans la deuxième illustration [fig. 2], ironiquement intitulée "Entrée en Conférence », c'est une "vue de la cuisine ${ }^{40}$ " qui est donnée par l'artiste, "à la façon hollandaise ${ }^{41}$ ", où curés et servantes s'affairent entre l'âtre et les fourneaux, au milieu de la vaisselle, des chaudrons et des casseroles, mais aussi des bouteilles et des victuailles. La spiritualité de la conférence est d'emblée hypothéquée par les "ragoûts odorants ${ }^{42}$ ". L'image suivante [fig. 3] représente " ces Messieurs au dessert »: "atteints [...] d'un commencement d'ébriétét3 ", les conférenciers s'apostrophent et se bagarrent, brisant la vaisselle, renversant les meubles et défenestrant le voisin. "La salle à manger ressemble à une cellule de Bicêtre dans laquelle on aurait enfermé dix poètes atteints d'aliénation mentale ", écrit Courbet ${ }^{44}$. La quatrième gravure [fig. 4] reprend Le Retour de la conférence de 1863, déjà décrit. Vient ensuite la cinquième planche [fig. 5], dont la légende indique qu'il s'agit d'un " autre mode de retour de la conférence". Ayant conduit à la foire " deux cochons gras dans sa charrette ", un paysan charge au retour " deux curés tellement gris, qu'ils ne peuvent faire un pas de plus ${ }^{45}$ ». Un cantonnier, témoin de la scène, "jette un coup d'œil satirique sur les curés, et dit au paysan : Tu n'as donc pas vendu ${ }^{46} \ldots$ »; la cochonnerie est ici hautement signifiante, retournant la réputation du réalisme et renvoyant à la lubricité des visions de saint Antoine et à l'iconographie satirique révolutionnaire du clergé gras ${ }^{47}$. La dernière composition [fig. 6], dans un registre stercoraire, montre "le coucher des conférenciers", que leurs gouvernantes tentent de soulager de leurs " débordements ${ }^{48}$ ». Chancelant, le curé s'écroule " en proie à des spasmes nerveux qui annoncent une prochaine catastrophe ${ }^{49}$ " et vomit dans un pot de chambre que lui tient sa servante.

38. Correspondance de Courbet, ouvr. cité, p. 184 (« aux jeunes artistes », 25 décembre 1861).

39. Les Curés en goguette, ouvr. cité, p. 8. On notera qu'en principe, ce n'était pas le curé qui sonnait les cloches, mais le sacristain. À des fins satiriques, Courbet procède ici à une inversion des rôles.

40. Ibid., p. 9.

41. Ibid., p. 10.

42. Ibid., p. 11.

43. Ibid., p. 15.

44. Ibid., p. 16.

45. Ibid., p. 29.

46. Ibid., p. 30.

47. Antoine de Baecque, La Caricature révolutionnaire, Paris, Presses du CNRS, 1988. Sur la survivance de cette iconographie du curé gras après la Révolution, voir Jacqueline Lalouette, La libre pensée en France, 1848-1940, Paris, Albin Michel, coll. « Bibliothèque histoire », 1997, p. 219-226.

48. Les Curés en goguette, ouvr. cité, p. 26.

49. Ibid., p. 26. 


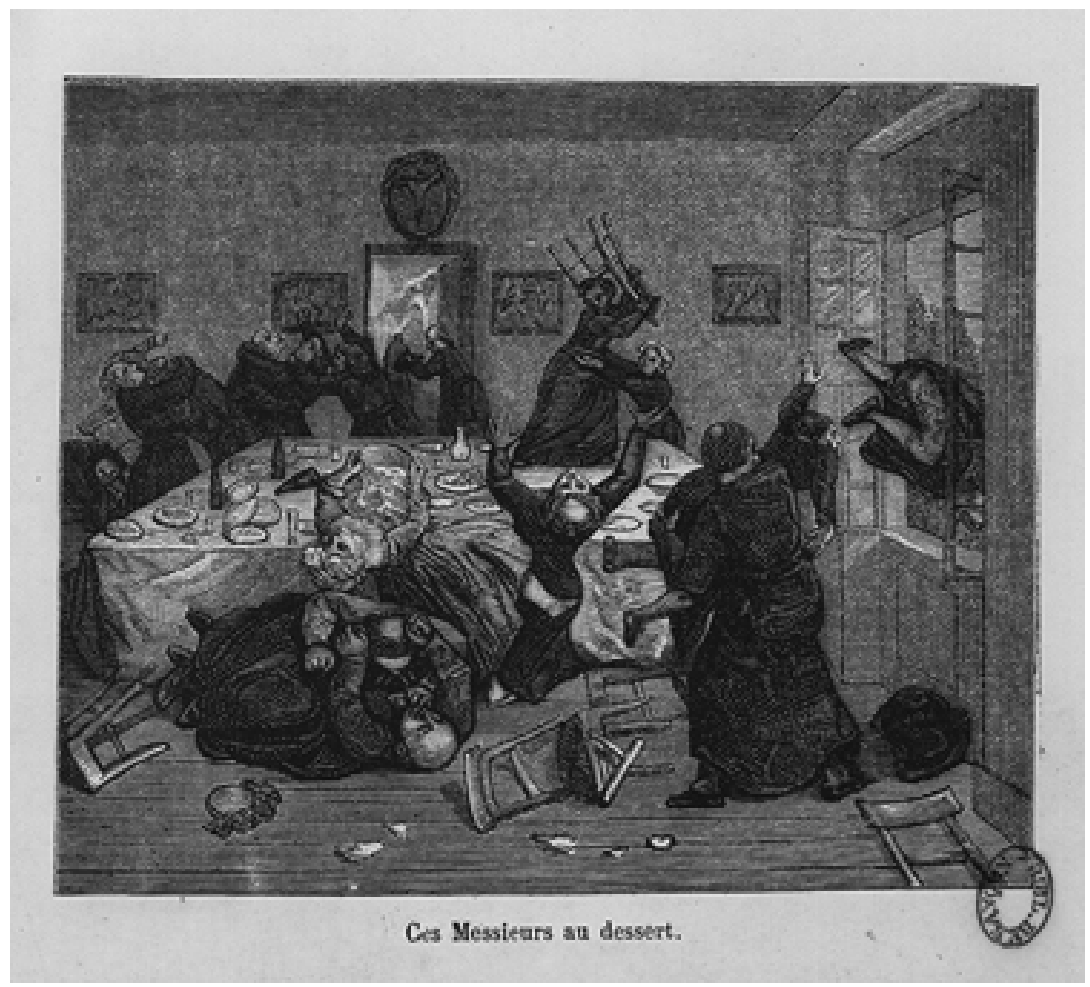

Figure 3. (C) Bibliothèque municipale de Besançon + BR.108.23.

\section{VÉRITÉ RÉALISTE VS IDÉALISME SOCIAL}

Par cette suite d'images, dont il faut souligner le dispositif narratif et le pouvoir de documentation du réalisme le plus cru - quand il n'est pas trivial -, Courbet entend "donner au public une idée des conférences ${ }^{50}$ ". En dénonçant leur véritable nature de "saturnales $^{51}$ " et d'orgies et l'absence de toute théologie, il prétend dévoiler la vérité des mœurs ecclésiastiques. Courbet qui, comme l'a rappelé Riat, avait peint sa pipe en signant "Courbet sans idéal et sans religion" ${ }^{52}$ ", ravale les ministres du culte catholique - « religion d'État ${ }^{53}$ " entravant " la liberté de conscience » et empêchant de "proclamer la liberté absolue des cultes ${ }^{54}$ " - au rang d'êtres aussi vils et charnels qu'ils se prétendent nobles et spirituels. Pour ce faire, il recourt au rire qualifié par Mikhaïl Bakhtine de "bas, matériel et corporel ${ }^{55}$ ", qui fonde son anticléricalisme et

50. Ibid., p. 30.

51. Ibid., p. 32.

52. Georges Riat, Gustave Courbet peintre, ouvr. cité, p. 203.

53. Courbet ne peut ignorer que le catholicisme n'est plus « religion d'État» depuis 1830, mais il feint de le croire pour les besoins de son pamphlet, dénonçant la collusion de l'Église et du Second Empire.

54. Les Curés en goguette, ouvr. cité, p. 32.

55. Mikhaïl Bakhtine, L'œuvre de François Rabelais et la culture populaire au Moyen Âge et sous la Renaissance, Paris, Gallimard, coll. «Bibliothèque des idées », 1970, p. 89. 
nourrit sa volonté de laïcisation de la vie sociale. Mais cette chronique illustrée des Curés en goguette, d'inspiration rabelaisienne - hantée d'ivresse, de bonne chère et de lubricité -, se double d'une critique de l'idéalisme social et esthétique telle que Proudhon y a été sensible :

Ce qu'a voulu montrer Courbet, à la façon des vrais artistes, c'est l'impuissance radicale de la discipline religieuse - ce qui revient à dire de la pensée idéaliste - à soutenir dans le prêtre la vertu sévère qu'on exige de lui : c'est que la perfection morale recherchée par la foi, par les œuvres de dévotion, par la contemplation d'un idéal mystique, se réduit à de lourdes chutes $[. . .]^{56}$.

Dans son commentaire du Retour de la conférence, Proudhon avait en effet souligné combien cette œuvre de Courbet ne devait pas être comprise comme " une mauvaise action " - n'étant ni un " outrage à la morale religieuse ", ni une " excitation au mépris d'une classe de citoyens ${ }^{57}$ "-, mais comme une dénonciation, à travers la figure du prêtre ivre et ridicule, de tout "adorateur de l'idéal et de l'absolu ${ }^{58}$ ", esclave de la "tyrannie de l'idéal"59".

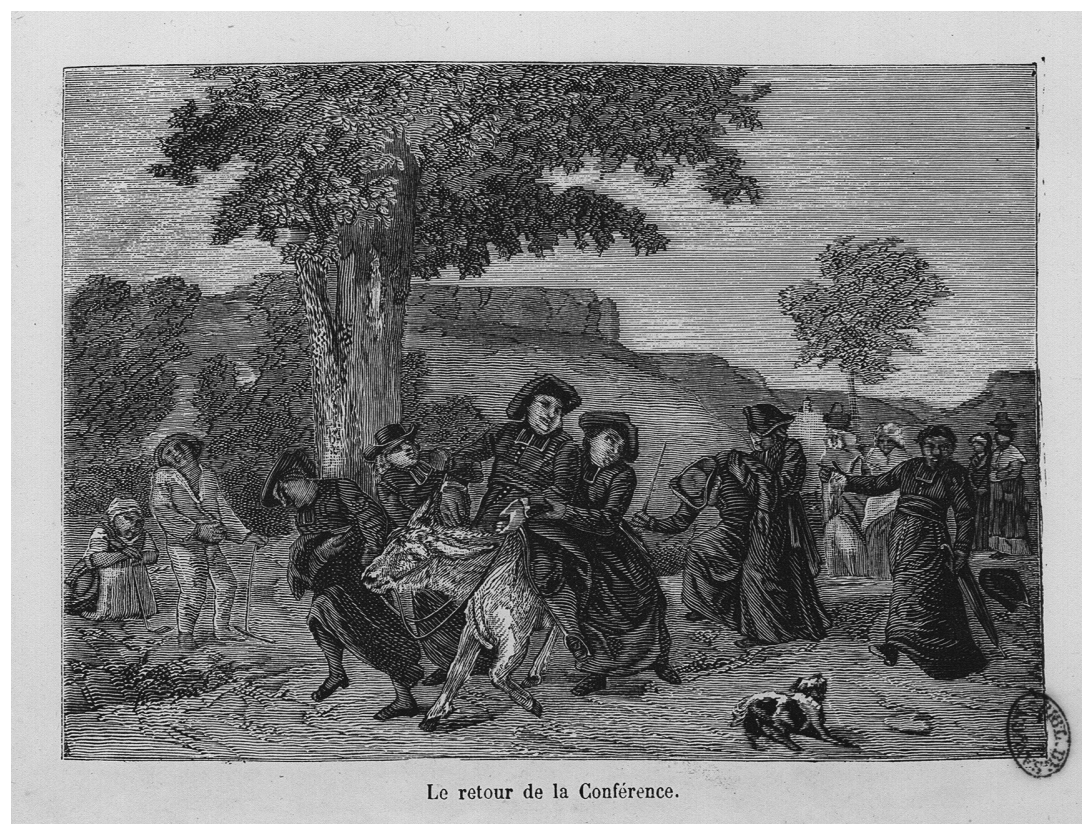

Figure 4. (C) Bibliothèque municipale de Besançon + BR.108.23.

56. Pierre-Joseph Proudhon, Du principe de l'art et de sa destination sociale, ouvr. cité, p. 164.

57. Ibid., p. 163.

58. Ibid., p. 164.

59. Ibid., p. 165. 
Proudhon s'inscrit en faux contre la lecture des critiques contemporains, parfois proches du peintre, qui n'avaient vu dans Le Retour de la conférence qu'une grosse farce ou une petite satire - au mieux la parodie d'une danse de dryades ou de Silène ivre -, un tableau bâclé, au "lâché si grave ", avec " des parties grossières, triviales, communes, et en même temps peintes en papier d'auberge ", selon les mots durs de Champfleury ${ }^{60}$. Théodore Duret est plus catégorique encore : en recourant à la caricature et au comique, Courbet « s'était laissé entraîner en dehors de sa sphère de pur artiste par des gens qui voulaient le tirer à eux ${ }^{61}$ ". Répondant à l'incompréhension rencontrée, jusque dans son propre camp, par son tableau de 1863, Courbet a donc voulu, avec ses illustrations ultérieures, "peindre au vif ${ }^{62}$ " la réalité humaine et sociale de la vie sacerdotale des campagnes, au moyen du réalisme dont il démontre la vertu salutaire postulée quelques années plus tôt : "car réaliste signifie ami sincère de la vraie véritée ${ }^{63}$ ". Du même coup, comme en différé, Courbet paraît répondre aux interrogations sur les origines de son tableau de 1863 : avait-il été le témoin de cet épisode - comme des Casseurs de pierres, dont il disait : "Je n'ai rien inventé, [...] chaque jour allant me promener, je voyais ces personnages ${ }^{64}{ }$ - ou l'avait-il fantasmé ? Cet incident était-il le fruit d'une réalité observée ou d'une imagination libérée en vue du combat anticlérical ? Selon Duret, « la scène n’a pas été vue, mais composée [...] et chargée dans une idée d'hostilité au clergé ${ }^{65}$ ". À l'inverse, Castagnary soutenait que le peintre avait vu la scène : «Il aimait la conter, la décrire avec force détails et le faisait toujours avec de grands éclats de rire ${ }^{66}$. " Par cette suite d'illustrations ultérieures, Courbet réaffirmait le pouvoir du réalisme et de sa charge expressive, aux confins de la satire et de la vérité, de la dénonciation et de la révélation. Rien d'étonnant à ce que le critique Thoré-Bürger se soit élevé contre l'exclusion du Retour de la conférence du Salon de 1863, "dans le pays de Rabelais, de Molière, de Voltaire et de Diderot, où Charlet, Raffet, Daumier, Henri Monnier n'ont pas ménagé leurs satires contre la bourgeoisie, la magistrature et le militaire ${ }^{67} "$. Courbet qui, depuis 1849 , avait l'ambition d' " encanailler l'art ${ }^{68}$ ", produisait ainsi une œuvre plus populaire que ne l'était sa peinture, malgré ses efforts, en apparentant explicitement ses dessins à l'imagerie simplifiée, élémentaire et volontiers malhabile à laquelle on assimilait ses tableaux, positivement ou pas ${ }^{69}$. Les images des Curés en goguette appartiennent au corpus des illustrations produites par Courbet pour Les Chansons populaires des provinces de France de Champfleury (1860), L'Histoire anecdotique des cafés et cabarets de Delvau (1862) ou Le Camp des bourgeois de Baudry (1868). Comme les chansons

60. Cité par Georges Riat, Gustave Courbet peintre, ouvr. cité, p. 207.

61. Théodore Duret, Courbet, Paris, Bernheim Jeune, 1918, p. 70.

62. Pierre-Joseph Proudhon, Du principe de l'art et de sa destination sociale, ouvr. cité, p. 166.

63. Correspondance de Courbet, ouvr. cité, p. 97 (au rédacteur en chef du Messager de l'Assemblée, 19 novembre 1851$)$.

64. Ibid., p. 82 (à Francis Wey, 26 novembre 1849).

65. Cité par Roger Bonniot, Gustave Courbet en Saintonge, ouvr. cité, p. 134 et 135.

66. Ibid., p. 135.

67. Cité par Georges Riat, Gustave Courbet peintre, ouvr. cité, p. 206.

68. Correspondance de Courbet, ouvr. cité, p. 82 (à Francis et Marie Wey, 26 novembre 1849).

69. Meyer Schapiro, « Courbet et l'imagerie populaire, Étude sur le réalisme et la naïveté », [19401941], Style, artiste et société, Paris, Gallimard, coll. « Tel », 2005, p. 273-328. 
à boire, joyeuses et viriles, triviales et grossières qu'il composa, ces illustrations sommaires empreintes de rusticité procèdent de la conscience qu'avait l'artiste d'être "provincial et plébéien ${ }^{70}$ ». Elles disent sa volonté de traduire les sentiments du peuple, par le biais d'un art fruste et démocratique - à la limite de l'" art sans art " qu'avait théorisé Champfleury à propos de la caricature ${ }^{71}$ - qui plaçait ses productions à proximité des assiettes peintes, gravures coloriées, chansons illustrées... dont il appréciait la naïveté, les imperfections et l'étrangeté.

\section{CONTRE LE CLERGÉ ET LES ULTRAMONTAINS}

Sans doute faut-il donc nuancer ce qu'avançait Meyer Schapiro, pour qui ces illustrations des Curés en goguette en disent moins sur les dogmes de l'Église que sur les croyances et le folklore superstitieux de la paysannerie à l'encontre du clergé comme classe sociale ${ }^{72}$. En effet, ces images cristallisent une attaque antireligieuse plus subtile, dont le tableau de 1863 portait déjà la trace et que le texte de la brochure de 1868 met en lumière : "Cette scène [d'ivrognerie ecclésiastique] se passait sous un arbre, où se trouvait une petite Notre-Dame en plâtre logée au fond d'une niche creusée dans le tronc, et protégée par un treillis de fil de fer. Cette niche était remplie d'un ex-voto et de menus bibelots à l'usage spécial des vieilles bigotes ${ }^{73}$. " À l'instar du chêne de Flagey peint par Courbet dans les mêmes années ${ }^{74}$, l'arbre que Proudhon décrira successivement comme un "chêne bénit » et un "vieux hêtre » a une signification majeure : c'est un $\pi \varepsilon \rho \iota \delta \varepsilon ́ \xi \iota \mathrm{o} \nu$, un arbre de vie qui symbolise le Christ ${ }^{75}$, autant qu'un arbre de la Liberté de tradition révolutionnaire - "monument républicain ", selon l'expression de Mona Ozouf ${ }^{76}$, ravivé par 1848 - au pied duquel dansent les curés ivres et blasphémateurs tant à son encontre qu'à celle de la statue votive. "La piété des populations, excitée par le clergé, a multiplié les croix et les images pieuses à travers les campagnes, au bord des chemins, dans tous les carrefours ${ }^{77}$ ": Proudhon a bien noté que cette scène est doublement sacrilège, car au-delà du mépris pour le culte des arbres ${ }^{78}$, elle montre la profanation du culte marial dans son exercice populaire, qu'atteste aussi le rire inextinguible du paysan à l'égard des curés profanateurs.

Par ses illustrations de 1868, Courbet élabore donc une satire anticléricale, selon le répertoire traditionnel et convenu, mais qu'il adapte au contexte politique du Second Empire. Depuis les guerres d'Italie (1859), le soutien des catholiques à Napoléon III n'est plus aussi net qu'aux premières années; Courbet ne s'en embarrasse pas. Il

70. Ibid., p. 290.

71. Bernard Vouilloux, Un art sans art, Champfleury et les arts mineurs, Lyon, Fage Éditions, 2009.

72. Meyer Schapiro, « Courbet et l'imagerie populaire... », ouvr. cité, p. 290.

73. Les Curés en goguette, ouvr. cité, p. 25.

74. Linda Nochlin, «Le Chêne de Flagey de Courbet : un motif de paysage et sa signification », 48/14, revue du musée d'Orsay, $\mathrm{n}^{\circ} 1,1989$, p. 15-25.

75. Chakè Matossian, Saturne et le sphinx..., ouvr. cité, p. 117.

76. Mona Ozouf, La Fête révolutionnaire, 1789-1799, Paris, Gallimard, 1976, p. 290.

77. Pierre-Joseph Proudhon, Du principe de l'art et de sa destination sociale, ouvr. cité, p. 168.

78. Sur le culte des arbres, voir Alain Corbin, La Douceur de l'ombre, Paris, Fayard, 2013. Voir aussi le catalogue de l'exposition Histoires d'arbres, Montreuil, Gourcuff Gradenigo, L'Isle-Adam, Musée d'art et d'histoire Louis Senlecq, 2012. 
maintient ses charges contre un régime qui a considérablement augmenté le nombre des paroisses et le budget du culte, qui accorde aux prêtres une sorte d'immunité en matière judiciaire ${ }^{79}$ et qui joue de la liturgie catholique pour donner au règne impérial une dimension spectaculaire. En condensant la vie des curés de campagne dans des épisodes scabreux et en les montrant jusque dans la déraison de leur ivresse profanatrice, Courbet s'attaque à un clergé auquel Napoléon III a octroyé ses faveurs comme à un pilier de l'ordre social. Par ses fêtes et ses processions, dont la monarchie de Juillet avait réduit l'ampleur et le nombre ${ }^{80}$, le catholicisme s'expose dans l'espace public; la construction de nouvelles églises pour les paroisses créées marque les paysages urbains et ruraux. Dans sa chronique illustrée marquée du sceau du réalisme, Courbet s'empare de cette visibilité nouvelle ou ravivée du catholicisme qui paraît vouloir effacer l'héritage révolutionnaire; il la retourne et en sape les fondements.

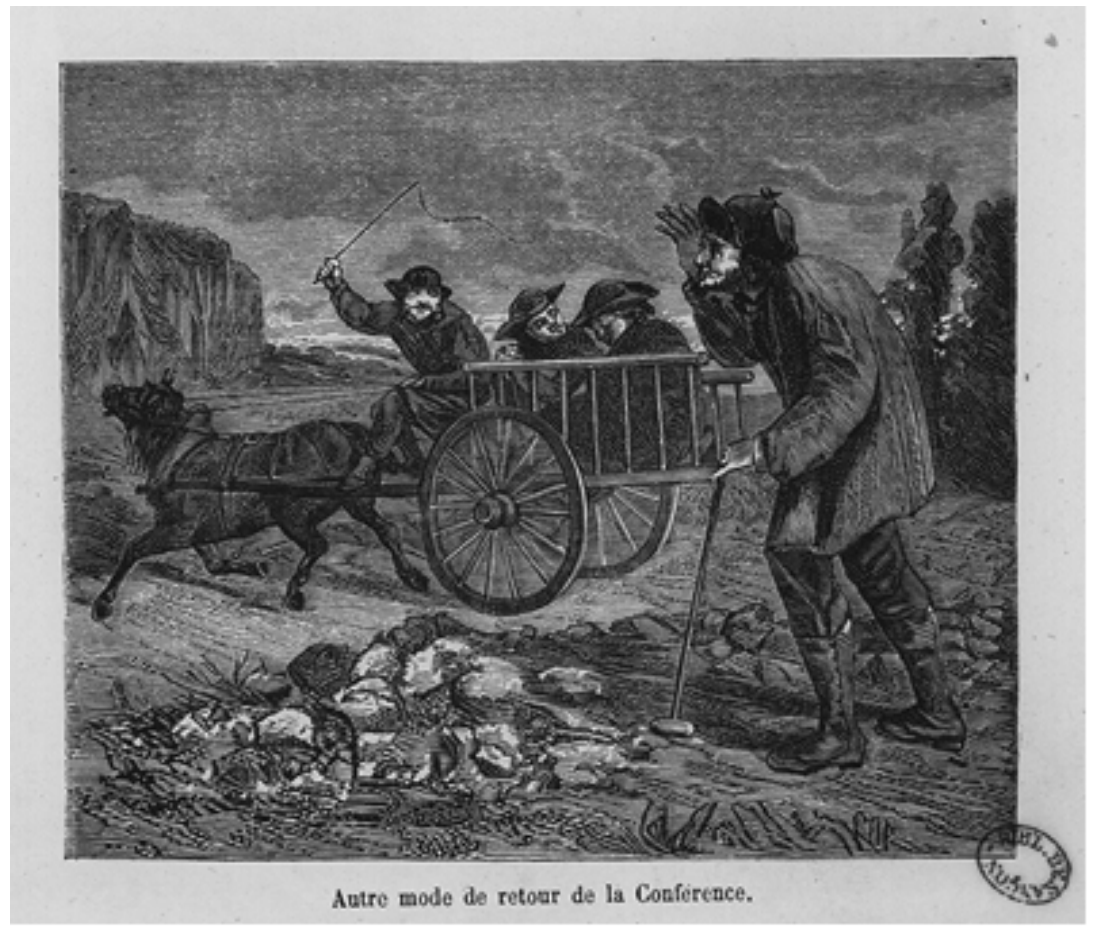

Figure 5. (C) Bibliothèque municipale de Besançon + BR.108.23.

Courbet s'attaque aussi aux ultramontains que leur aveu du pouvoir absolu du pape rend réfractaires à la modernité politique et sociale, alors que se développent les

79. Jacques-Olivier Boudon, Religion et politique en France depuis 1789, Paris, Armand Colin, coll. « Cursus », 2007, p. 81-82.

80. Voir Paul d'Hollander, « Processions et liberté sous la monarchie de Juillet », dans Paul d'Hollander (dir.), L'Église dans la rue, Les cérémonies extérieures du culte en France au XIXe siècle, Limoges, PULIM, 2001, p. 57-67. 
courants rationalistes ou positivistes et tandis que naissent les premiers groupes de Libre-Pensée aspirant à la destruction des religions ${ }^{81}$. À cet égard, l'artiste participe de ce mouvement de laïcisation de la société, qui amorce une action militante structurée contre l'Église. Le tableau du Retour de la conférence, comme sa version gravée, évoque la dévotion mariale en représentant une statuette de la Vierge dans une niche arboricole. Sans doute faut-il y voir une allusion à une pratique populaire très répandue en Franche-Comté ${ }^{82}$. Mais peut-être s'agit-il aussi, plus particulièrement, d'une référence aux événements de Scey-Maisières, près d'Ornans. Le 3 avril 1803, dimanche des Rameaux, Cécile Mille, se hâtant vers l'église où elle devait faire sa communion, eut une apparition ; la Vierge lui désigna un chêne. La fillette ne comprit pas, mais quelques mois plus tard, le 13 août, au même endroit, son père vit deux lumières brillantes. Il ouvrit l'écorce du tronc et trouva une vieille statuette, datant du temps de Louis XIV, représentant la Vierge à l'Enfant. C'est pourquoi, en juillet 1863, fut posée, non loin de là, la première pierre d'une chapelle. Ce monument néo-gothique dû à l'architecte Pierre Bossan fut inauguré en août 1869, sous le vocable de Notre-Dame du Chêne. En plaçant cet élément du culte marial au centre d'une scène anticléricale, Courbet vise l'attitude blasphématoire des curés ivres, mais stigmatise aussi l'activisme ultramontain qui a dénaturé la piété mariale populaire en dévotion démonstrative ${ }^{83}$, avec processions, couronnements, sanctuaires et statues monumentales - au Puy, à Biarritz, Marseille ou Monton ${ }^{84}$. À Maisières, le jour de l'Assomption, les villageois s'étaient longtemps retrouvés autour du chêne ; son tronc était ouvert par un vannier ; il en extrayait la statue de la Vierge, qui, placée en pleine lumière, apparaissait éblouissante. La construction de la chapelle fit disparaitre cette cérémonie, altérant ainsi le " religieux naturel » fait de « coutumes intimement mêlées à la vie quotidienne ${ }^{85}$ ". Courbet montre dans ses prêtres les profanateurs d'une dévotion populaire qu'ils ignorent, et les réduit à l'état de ministres d'une foi débarrassée de tout recueillement, extériorisée et festive, que leur ivresse tourne en farce grotesque.

Le Retour de la conférence de 1863 et le cycle des illustrations des Curés en goguette de 1868 constituent donc moins des caricatures anticléricales égarées dans la peinture, que de véritables pages critiques à l'égard de la politique et de l'idéologie du Second Empire - en l'occurrence à travers ses alliances religieuses. Deux actions contemporaines de Courbet l'attestent par ailleurs : Le Chêne de Flagey, appelé chêne de Vercingétorix, Camp de César près d'Alésia, Franche-Comté (1864, Ornans, musée

81. Jacqueline Lalouette, La libre pensée en France..., ouvr. cité.

82. Pierre Irigoin, Les Oratoires de France depuis les origines, Aix-en-Provence, Éditions des Amis des oratoires, 1977, p. 291-319. Paul Dieudé (dans Les Oratoires du Doubs, Aix-en-Provence, Éditions des Amis des oratoires, 1998, p. 19) a répertorié 21 niches arboricoles dans le seul département du Doubs.

83. Sur ce phénomène, voir Gérard Cholvy et Yves-Marie Hilaire, Histoire religieuse de la France contemporaine, 1800-1880, Toulouse, Privat, coll. « Bibliothèque historique », 1985, vol. 1, p. 176 et suiv.

84. Voir Anne Pingeot, «Les Vierges colossales du Second Empire », dans le catalogue d'exposition La Sculpture française au XIX $X^{e}$ siècle, Paris, Grand Palais, 1986, p. 208-212. Voir aussi Paul d'Hollander et Claude Langlois (dir.), Foules catholiques et régulation romaine. Les couronnements de vierges de pèlerinage à l'époque contemporaine (XIX ${ }^{e}$ et XX $X^{e}$ siècles), Limoges, PULIM, 2012.

85. Gérard Cholvy et Yves-Marie Hilaire, Histoire religieuse de la France contemporaine..., ouvr. cité, p. 102-103. 


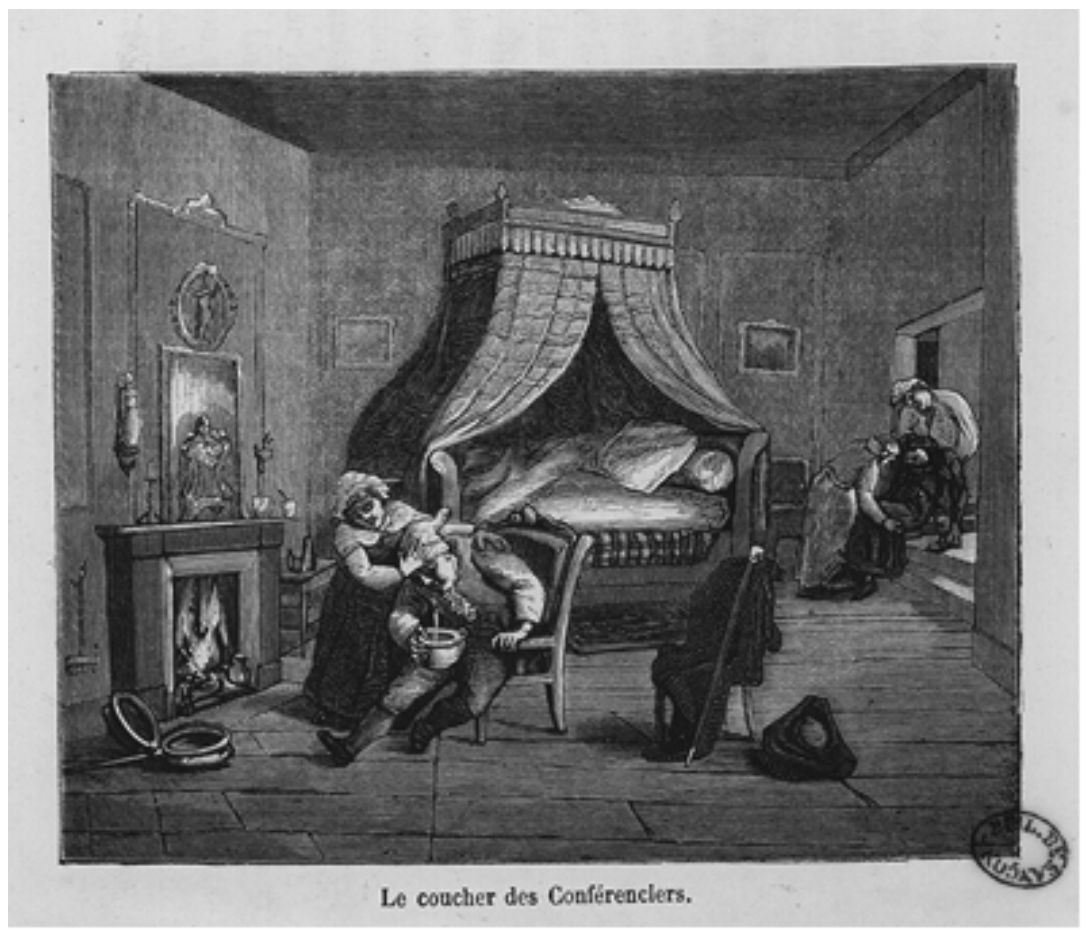

Figure 6. (C) Bibliothèque municipale de Besançon + BR.108.23.

Courbet), par lequel Courbet entre dans la querelle relative à la localisation d'Alésia en prenant parti contre le choix bourguignon de Napoléon III ; Opinions et propos d'un citoyen d'Ornans ${ }^{86}$, pamphlet où Courbet met en scène un incident survenu quelques années plus tôt à Ornans, entre le maire bonapartiste Bidalot et la chorale libérale d'Urbain Cuenot. Dans les deux cas, comme dans ses scènes de curés en conférence arrosée, Courbet reproche à l'administration impériale son autoritarisme et son interventionnisme stériles contre les initiatives populaires locales - seules garantes de la vie démocratique. C’est ce qu'il disait en 1868 à Castagnary ${ }^{87}:$ « [...] les corps établis, les académies de toutes sortes, la gouvernementation autoritaire dénotent un état de choses faux et l'entrave du progrès. »

(Université de Bourgogne)

86. Ce texte de 1868 est reproduit dans le Bulletin des Amis de Gustave Courbet, $\mathrm{n}^{\circ}$ 51, 1974, p. 11-23.

87. Correspondance de Courbet, ouvr. cité, p. 306-307 (à Jules Castagnary, 17 octobre 1868). 